\title{
PSMA-targeting iron oxide magnetic nanoparticles enhance MRI of preclinical prostate cancer
}

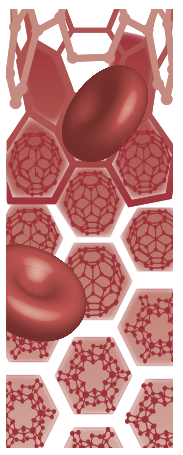

\begin{abstract}
Aim: To evaluate the potential of newly-developed, biocompatible iron oxide magnetic nanoparticles (MNPs) conjugated with J591, an antibody to an extracellular epitope of PSMA, to enhance MRI of prostate cancer. Materials \& methods: Specific binding to PSMA by J591-MNP was investigated in vitro. MRI studies were performed on orthotopic tumor-bearing NOD.SCID mice $2 \mathrm{~h}$ and $24 \mathrm{~h}$ after intravenous injection of J591-MNPs, or non-targeting MNPs. Results \& conclusion: In vitro, MNPs did not affect prostate cancer cell viability, and conjugation to J591 did not compromise antibody specificity and enhanced cellular iron uptake. Magnetic resonance contrast of tumors was increased in vivo using PSMA-targeting MNPs, but not by non-targeting MNPs. This provides proof-of-concept that PSMA-targeting MNPs have potential to enhance magnetic resonance detection/localization of prostate cancer.
\end{abstract}

Keywords: cancer imaging • iron oxide magnetic nanoparticles (MNPs) • MRI • prostate cancer $\bullet$ prostate specific membrane antigen (PSMA) • targeted imaging $\bullet$ targeted

nanoparticles

\section{Background}

MRI is a useful imaging tool in prostate cancer management. It provides excellent soft tissue contrast and multidimensional information, does not involve exposure to ionizing radiation, and is non-invasive [1]. However, like other imaging modalities such as computed tomography (CT), transurethral ultrasound (TRUS) and nuclear imaging, MRI cannot adequately detect small tumors [2]. Improvements in tumor-imaging technologies are urgently required for early detection of disease, staging, and/or real-time assessment of response to therapy in prostate cancer patients. Iron oxide magnetic nanoparticles (MNPs) have emerged as powerful contrast agents for MRI [3]. Their superparamagnetic properties make them effective at reducing transverse (spin-spin) T2-relaxation time, causing negative contrast in magnetic resonance (MR) images [4,5]. MNP-assisted MRI has the potential to improve the assessment of cell surface receptor expression on tumors, liver function (macrophage content and activity), inflammation, degenerative diseases, angiogenesis, perfusion and apoptosis [6]. Currently, certain iron oxide-based MNPs have been approved for use in clinical MRI, for instance ferumoxil (GastroMARK) enhances imaging of the bowel. In this study, the potential ability of MNPs to enhance MRI of prostate cancer is evaluated. MNPs brane antigen (PSMA)-targeting antibody, J591, via a 1,2-distearoyl-sn-glycero-3-phosphoethanolamine- $\mathrm{N}$-[amino (polyethylene glycol) (PEG-DSPE) linkage, for delivery of the nanoparticle cargo to prostate cancer cells. PSMA, a 750-amino acid, type 2 transmembrane glycoprotein with folate hydrolase or carboxy peptidase II activity [7], is an ideal molecular target for imaging for a number of reasons. While PSMA is expressed in normal prostate epithelial and benign hyperplastic cells, it exists as a truncated form (PSM') through alternative splicing and is found in the cytosol, whereas a transmembane form is expressed at high levels in prostate cancer [8]. are conjugated to the prostate specific mem-
Brian Wan-Chi Tse', Gary J Cowin'2, Carolina Soekmadji', Lidija Jovanovic', Raja S Vasireddy', Ming-Tat Ling', Aparajita Khatri ${ }^{3}$, Tianqing Liu $^{4}$, Benjamin Thierry ${ }^{4}$

\& Pamela J Russell $*, 1$

${ }^{1}$ Australian Prostate Cancer Research Centre-Queensland, Institute of Health \& Biomedical Innovation, Queensland University of Technology, Translational Research Institute, Brisbane, Queensland, Australia

${ }^{2}$ National Imaging Facility, Centre for Advanced Imaging, University of Queensland, Brisbane, Queensland, Australia

${ }^{3}$ Ceramisphere Pty Ltd (Health Care Division), Sydney, New South Wales, Australia

${ }^{4}$ Ian Wark Institute, University of South Australia, Adelaide, South Australia, Australia

*Author for correspondence:

Tel.: +61734437240

Fax: +6173176 7440

pamela.russell@qut.edu.au 
The PSMA/PSM' mRNA ratio is lowest in normal tissue and increases with increasing Gleason score [9]. PSMA expression is upregulated in prostate cancer, including lymph node and bone metastases, upregulated in androgen deprivation conditions, and elevated in late stage castrate-resistant prostate cancer (CRPC) [10-12]. In addition, PSMA has a cytoplasmic domain that contains a novel and unique amino acid sequence of MXXXL that mediates its internalization and endosomal recycling [13,14], which can lead to high intracellular retention of PSMA-targeting biomaterials such as MNPs. J591 was selected as the PSMA-targeting agent due to its well-established specificity. ${ }^{177} \mathrm{Lu}-\mathrm{J} 591$ has previously been shown to target all known sites of disease in all treated prostate cancer subjects [15], and in another study, treatment with ${ }^{111} \mathrm{In}-\mathrm{J} 591$ and then ${ }^{90} \mathrm{Y}-\mathrm{J} 591$ revealed $89 \%$ of known bony metastases and $69 \%$ of soft tissue lesions [16]. In this study, we provide proof-of-concept that J591-labeled MNPs can enhance MRI of orthotopic xenograft prostate cancer in mice.

\section{Materials \& methods}

Preparation \& characterization of magnetic nanoparticles

Monocrystalline iron oxide nanoparticles were synthesized using a small modification of the solventfree one-pot procedure initially reported by Niederberger et al. [17,18]. Briefly, $\mathrm{Fe}$ (acac) 3 powder $(500 \mathrm{mg})$ was mixed with benzyl alcohol $(10 \mathrm{ml})$ and sealed in a Teflon cup. The mixture was placed and tightly sealed in a steel container and transferred in a furnace at $175^{\circ} \mathrm{C}$ for $48 \mathrm{~h}$. A ligand exchange procedure with oleic acid was used to hydrophobise the synthesized nanoparticles. The nanoparticle suspension in benzyl alcohol was centrifuged once and the pellet resuspended in chloroform $(5 \mathrm{ml})$. Oleic acid was then directly added in excess $(50 \mu \mathrm{l})$ into the nanoparticle solution, which was then immediately placed in a sonic bath for $60 \mathrm{~min}$. The oleic acid-capped nanoparticles were washed three times using high speed centrifugation to remove excess oleic acid. The oleic acid-capped nanoparticles were used as solid templates for the formation of core-shell micellar hybrid structures. Typically, $1 \mathrm{ml}$ of a mixture of 1,2-distearoyl-sn-glycero3-phosphoethanolamine-N-[carboxyl (polyethylene glycol)] (DSPCE-PEG-COOH), 1,2-dimyristoyl-snglycero-3-phosphoethanolamine-polyethylene glycol (DSPE-PEG) and L-alpha-phosphatidylcholine (PC) (20:50:30\% weight $2 \mathrm{mg} / \mathrm{ml}$ ) in chloroform was prepared and mixed with the oleic acid capped-nanoparticles $(-10 \mu \mathrm{mol} \mathrm{Fe})$. To facilitate monitoring of cellular interactions, the lipophilic near-infrared fluorescence probe $1,1^{\prime}$-dioctadecyl-3,3,3',3'-tetramethyl indotricarbocyanine Iodide (DIR) was added to the phos- pholipid mixture $(0.5 \%$ weight $)$. The solvent was evaporated at $55^{\circ} \mathrm{C}$ and the samples were stored under nitrogen at $-20^{\circ} \mathrm{C}$ until used. The dry nanoparticle film was heated to $70^{\circ} \mathrm{C}$ and rehydrated with $1 \mathrm{ml}$ $\mathrm{H}_{2} \mathrm{O}$. Empty micelles were removed by a round of centrifugation at $14000 \mathrm{rpm}$ and the pellet was resuspended in $\mathrm{H}_{2} \mathrm{O}$. To enable conjugation with the J591 monoclonal antibodies, the terminal carboxyl groups of the DSPCE-PEG-COOH were activated using N-hydroxysuccinimide (NHS, $50 \mathrm{mM}$ ) and $\mathrm{N}$-(3dimethylaminopropyl)-N'-ethylcarbodiimide hydrochloride (EDC, $100 \mathrm{mM}$ ) for $10 \mathrm{~min}$. The nanoparticle suspensions were centrifuged and resuspended into $1 \mathrm{ml}$ of phosphate buffered saline (PBS) and quickly added to the antibody solution (75 $\mu$ g antibody per $10 \mu \mathrm{mol} \mathrm{Fe}$ ). The mixture was left to react overnight after which the antibody conjugated nanoparticles were purified by centrifugation at $4^{\circ} \mathrm{C}$. Hydrodynamic diameters and polydispersity indexes were determined using a Zetasizer Nano ZS equipped with a $633 \mathrm{~nm}$ $\mathrm{He}-\mathrm{Ne}$ laser (Malvern Instruments). Transmission electron microscopy measurements were conducted using a Philips CM100 transmission electron microscope.

\section{Cell lines}

The human prostate cancer cell lines LNCaP, PC3, DU145, 22RV1 (all sourced from American Type Culture Collection [ATCC], USA), BPH-1 (from S Hayward, Vanderbilt University Medical Center, USA), LNCaP-C42B (abbreviated C42B) (from L Chung, Cedars-Sinai Medical Center, USA) [19,20], LNCaPLN3 (from C Pettaway, MD Anderson Cancer Center, University of Texas, USA) and LNCaP-luc (generated in-house) were maintained in Roswell Park Memorial Institute (RPMI) media containing 5\% fetal bovine serum (FBS) . LNCaP-luc cells were generated using the Viralpower Lentiviral gene expression system (Invitrogen, USA) according to the manufacturer's instructions. Briefly, supernatant containing the lentivirus was mixed with polybrene $(8 \mu \mathrm{g} / \mathrm{ml})$ and used to infect LNCaP cells. After infection, positive transfectants were selected as a pool by treatment with blasticidine $(8 \mu \mathrm{g} / \mathrm{ml})$ for 6 days. RWPE-1 cells (ATCC) were grown in keratinocyte serum-free media supplemented with recombinant human epidermal growth factor $(5$ $\mathrm{ng} / \mathrm{ml}$ final concentration) and bovine pituitary extract $(50 \mathrm{ng} / \mathrm{ml})$. The J591 hybridoma (from N Bander, Weill Medical Centre, Cornell University, USA) which produces the anti-PSMA antibody [21], was maintained in Hybridoma Serum-Free Media (SFM). All media and supplements were sourced from Gibco, Life Technologies. All cell lines were incubated at $37^{\circ} \mathrm{C}$ in a humidified atmosphere of $5 \% \mathrm{CO}_{2} /$ air. 


\section{Purification of anti-PSMA antibody (J591) by affinity chromatography}

The hybridoma J591 was cultured as described until the media were exhausted, and the secreted antibody was harvested. The hydridoma culture supernatant was passed through a HiTrap Protein HP column (GE Healthcare, UK) according to the manufacturer's instructions, on an AKTA FPLC system (GE Healthcare, UK). The bound $\operatorname{IgG}$ was eluted using $0.1 \mathrm{M}$ glycine, $\mathrm{pH} 3$, and the $\mathrm{pH}$ was immediately neutralized with $1 \mathrm{M}$ Tris-base, $\mathrm{pH}$ 9.0. The eluates were dialyzed against PBS and the purity of antibody preparations was determined by SDS-PAGE followed by Coomassie blue (Biorad, USA) staining. The concentration was determined by BCA protein assay (Pierce Biotechnology, USA).

\section{Flow cytometric detection of PSMA}

Cells were grown to $80 \%$ confluence in T75 flasks, washed twice in PBS then lifted using non-enzymatic cell dissociation buffer (Sigma Aldrich, USA). Cells were then washed in PBS containing 5\% FBS (FACSWASH) then resuspended at $10^{6} \mathrm{cells} / \mathrm{ml}$. One hundred $\mu \mathrm{l}$ of cell suspension was incubated with affinitypurified J591 antibody on ice for $1 \mathrm{~h}$, and then washed thrice in FACSWASH. Cells were then incubated with a secondary AlexaFluor 488-labelled donkey anti-mouse IgG $(\mathrm{H}+\mathrm{L})$ antibody (Life Technologies, USA) for $30 \mathrm{~min}$ on ice, before washing thrice in FACSWASH. Cells were then run on a FACS Canto (BD Bioscience, USA) and data analyzed using Kaluza software (Beckman Coulter, USA).

\section{Cell toxicity assays}

The effect of MNPs on the viability of prostate cancer cells was determined using Alamar blue reagent (Invitrogen, Life Technologies). Cells were seeded at 5000 cells per well (100 $\mu$ l volume) in a 96-well plate, and cultured overnight in growth media. Various amounts of MNPs in $100 \mu \mathrm{l}$ volume were added to the wells (see relevant figure legend for amounts), and plates were returned to the incubator for 48 h. $20 \mu \mathrm{l}$ of Alamar blue reagent was added to all wells, which were then incubated at $37^{\circ} \mathrm{C}$ for 3 to $4 \mathrm{~h} .100 \mu \mathrm{l}$ of supernatant was transferred to a black 96-well plate, and fluorescence at $590 \mathrm{~nm}$ was measured using a FLUOstar Omega plate reader (BMG Labtech, Germany).

\section{Prussian blue staining for uptake of MNPs}

For in vitro studies, cells grown on chamberslides or on cover slips were incubated for $2 \mathrm{~h}$ at room temperature with MNP alone, MNPs conjugated to J591 (J591-MNP) or J591 alone. Cells were fixed in 2\% paraformaldehyde, washed with PBS, and then stained for iron using the Accustain reagent (Sigma Aldrich, USA) according to the manufacturer's instructions. Briefly, the cells were stained in Iron Staining solution for $10 \mathrm{~min}$, rinsed in de-ionized water, and then counterstained for $5 \mathrm{~min}$ in Pararosaniline solution. The cells were rinsed in de-ionized water again, air-dried, then mounted with a coverslip. For in vivo studies, all mice were euthanized immediately after MRI (approximately $24 \mathrm{~h}$ post-MNP injection), and the harvested tissues were fixed in $10 \%$ neutral buffered formalin for 48 to $72 \mathrm{~h}$ and then in $70 \%$ ethanol for a further $48 \mathrm{~h}$. The tissues were then processed on a Tissue-Tek VIP6 tissue processor, blocked in paraffin then sectioned at $5 \mu \mathrm{m}$ thickness. The same Accustain protocol was used to perform iron staining of tissue sections.

\section{Fluorescence microscopy}

C42B cells grown on coverslips were incubated for $2 \mathrm{~h}$ at room temperature in the presence of MNPs alone, J591-MNP or J591 alone. Cells were washed, incubated with AlexaFluor-488 donkey anti-mouse antibody (Life Technologies, USA) for $30 \mathrm{~min}$ at room temperature, washed in PBS and then fixed in 2\% paraformaldehyde. Cells were then washed once with PBS, and coverslips were mounted onto slides. Images were acquired on a Zeiss confocal microscope.

\section{Animal studies (orthotopic prostate xenograft model)}

All studies were in accordance with guidelines of the Animal Ethics Committees of Queensland University of Technology and The University of Queensland, and the Australian Code for the Care and Use of Animals for Scientific Purposes. LNCaP or bioluminescent LNCaP-luc cells were lifted from culture flasks with trypsin, washed twice with PBS and resuspended in PBS. Cell counts were performed with a hemocytometer and viability was assessed by trypan blue exclusion. For intraprostatic injections, hair was removed from the abdomen of mice using a hair clipper, and the mice were anesthetized with ketamine $(25 \mathrm{mg} / \mathrm{kg})$ and xylazine $(5 \mathrm{mg} / \mathrm{kg})$, and received buprenorphine $(0.1 \mathrm{mg} / \mathrm{kg})$ for analgesia. An incision at the midline of the abdomen of NOD/SCID mice was made through the skin, exposing the dorsal prostate. $1 \times 10^{6} \mathrm{LNCaP}$ luc cells were injected into the dorsal prostate in $25 \mu \mathrm{l}$ volume. The bladder was returned to the abdomen and the incision closed with sutures. Tumor development was monitored by bioluminescence imaging using an IVIS Spectrum (Xenogen, USA) weekly for a total of 4 weeks after tumor cell implantation. For bioluminescence imaging, mice were injected intraperitoneally with D-luciferin diluted in PBS (15 $\mathrm{mg} / \mathrm{ml}$ stock) at $150 \mathrm{mg} / \mathrm{kg}$. Mice were anaesthetized via isoflurane 
inhalation, and imaged 8-12 min after injection with D-luciferin. Bioluminescence was analyzed using Living Imagine software (Xenogen, USA).

\section{MRI studies (pilot studies)}

In experiments involving MRI on non-tumor-bearing mice injected with MNPs directly into the prostate, mice were first anesthetized with ketamine and xylazine. A small midline incision was made on the abdomen of NOD/SCID mice, exposing the dorsal prostate, and $12 \mu \mathrm{g}$ of MNPs in $20 \mu \mathrm{l}$ volume was injected into it. MRI was performed $1 \mathrm{~h}, 24 \mathrm{~h}$ and $48 \mathrm{~h}$ thereafter. In experiments involving MRI on mice bearing orthotopic $\mathrm{LNCaP}$ or LNCaP-luc tumors, mice were injected with MNPs alone ( $\mathrm{n}=2)$ or J591-MNP $(\mathrm{n}=3)$ intravenously via tail vein at $6 \mathrm{mg} / \mathrm{kg}$ and MRI was performed $2 \mathrm{~h}$ and $24 \mathrm{~h}$ thereafter. This dosage was based on similar studies that also involved tail vein injection of MNPs to enhance MRI of other cancer types in rodents [22-24]. The wellbeing of all mice throughout the duration of the experiments, particularly after MNP administration, was closely monitored. The animal welfare assessment methodology by DB Morton was applied [25]. All mice were euthanized by $\mathrm{CO}_{2}$ asphyxiation immediately after their final MRI scan ( $24 \mathrm{~h}$ or $48 \mathrm{~h}$, depending on experiment; see figure legends). Mice were imaged on a Bruker (Germany) AV700 MRI system consisting of a $16.4 \mathrm{~T}$ vertical magnet interfaced to an AVANCE II spectrometer running Paravision 5 using a $25 \mathrm{~mm}$ volume coil in a micro 2.5 gradient set, under isofluorane anesthesia. Recirculating water in the gradient set was maintained at $30^{\circ} \mathrm{C}$. A series of fast low-resolution gradient echo images were acquired as localizers for placement of the following image sets. A series of axial gradient images, centered on the prostate, were acquired with typical parameters as follows: repetition time $(\mathrm{TR})=$ $252 \mathrm{~ms}$, echo time $(\mathrm{TE})=2.8 \mathrm{~ms}$, pulse angle $30^{\circ}$, fieldof-view $=30 \times 30 \mathrm{~mm}$, matrix $=320 \times 320$, slice thickness $=0.8 \mathrm{~mm}$, slice gap $=0 \mathrm{~mm}$, number of slices $=19$, numbers of averages $=12$, acquisition time $=16 \mathrm{~min}$.

\section{Results}

Characterization of MNPs \& J591 alone

The MNP preparation was developed based on the solvent-evaporation method initially reported for the preparation of quantum dots phospholipid micellar hybrids [26]. Highly monocrystalline magnetite nanoparticles were synthesized using the one-pot benzyl alcohol route described by Niederberger et al. and further phase-transferred into chloroform using oleic acid as a capping agent. The oleic acid-coated nanoparticles were then mixed with PEGylated phospholipids and the solvent evaporated. Rehydration of the dry film yielded the phospholipid-coated MNPs which could be easily purified from empty micelles using centrifugation. Terminal carboxylic groups on the 1,2-distearoyl-sn-glycero-3-phosphoethanolamine- $\mathrm{N}$ - [carboxyl (polyethylene glycol)] were activated using EDC/NHS and further reacted with the J591 antibody to yield the immune-targeted MNPs. Characterization of the nanoparticles was carried on using transmission electron microscopy and dynamic light scattering measurements which showed that the sample consisted of small clusters of the MNPs (Figure 1A). The mean hydrodynamic diameter of the MNP clusters was $110 \mathrm{~nm}$ and the polydispersity index 0.127 as determined using dynamic light scattering (Figure 1B). Affinity-purified J591 antibody was shown to be specific for PSMA through binding only to known PSMA-positive cell lines (22RV1, LNCaP, and C42B cells) but not to those that are PSMA-negative (BPH-1, RWPE-1 and DU145 cells) as determined by flow cytometry (Figure 1C).

\section{MNPs are non-toxic to prostate cancer cells}

To determine if MNPs have any toxic effects on cells, RWPE-1 cells (non-tumorigenic prostate cell line), LNCaP, C42B, PC3 and DU145 cells (tumorigenic cell lines) were incubated with various doses of MNPs alone for $48 \mathrm{~h}$. The viability of cells was not affected, as assessed by Alamar blue reagent (Figure 2).

\section{J591-MNP led to increased tumor uptake of iron \& binding of J591 to PSMA is not compromised when conjugated to MNPs} To assess whether or not conjugation of MNPs with J591 facilitates iron uptake by tumors in vitro, C42B cells (PSMA-positive) were incubated with MNP alone or J591-MNP. Confocal microscopy shows minimal iron uptake in cells treated with MNPs alone, but significant uptake was seen with J591-MNP (Figure 3A). The J591 component of J591-MNP could also be visualized with the FITC-labeled anti-mouse secondary antibody. Similarly, Prussian blue staining showed greater iron uptake by LNCaP-LN3 cells (PSMApositive) in vitro when incubated with J591-MNP than with MNP alone (Figure $3 \mathrm{~B}$; top panel), confirming that conjugation of MNPs to J591 facilitated their uptake by the prostate cancer cells. The immunohistochemical staining intensity for PSMA by J591-MNP was similar to that of J591 alone, indicating that conjugation of MNP did not compromise antibody binding to PSMA (Figure 3B; bottom panel).

\section{Direct injection of MNPs into the prostate of} mice induces negative contrast on MR images To provide proof-of-concept that the presence of MNPs within the prostate is detectable by MRI, non tumor-bearing mice were injected with MNPs alone 
directly into their normal prostate. MR images taken $1 \mathrm{~h}$ after MNP injection showed significant darkening at that anatomical site. A similar degree of negative contrast was seen in the same mouse on MRI images taken $24 \mathrm{~h}$ and $48 \mathrm{~h}$ post-MNP injection (Figure 4). The darkening effect as a result of the MNP facilitated the visualization and localization of the prostate.

\section{Enhanced MRI of orthotopic xenograft LNCaP tumors by J591-MNP (pilot study)}

To evaluate the potential of PSMA-targeting MNPs as an imaging agent for MRI of prostate cancer, MRI was performed on mice with pre-established orthotopic LNCaP-luc tumors and intravenously injected with either MNPs alone or J591-MNPs. MR images of tumors from mice that received the J591-MNP conjugates showed significant darkening at the prostate region, at the 2- and 24-h post-injection timepoints (Figure 5). Negligible darkening effect was observed in mice that received intravenous MNPs alone (Figure 5). Prussian blue staining of resected tumors from J591MNP mice showed significant accumulation of iron within the tumor. In contrast, little iron accumulation was seen in the tumors from the MNP alone group
(A)

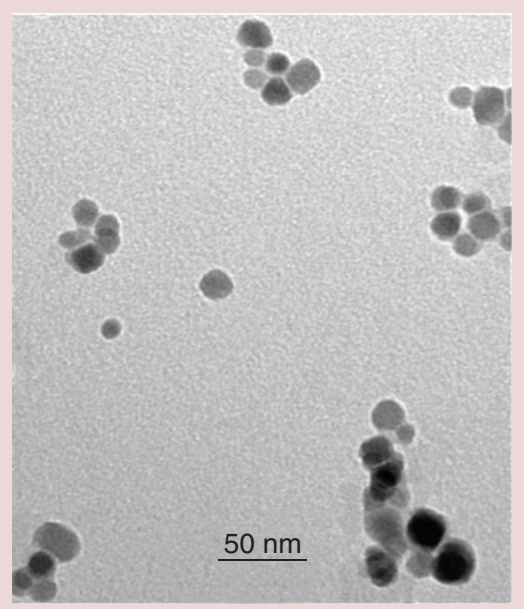

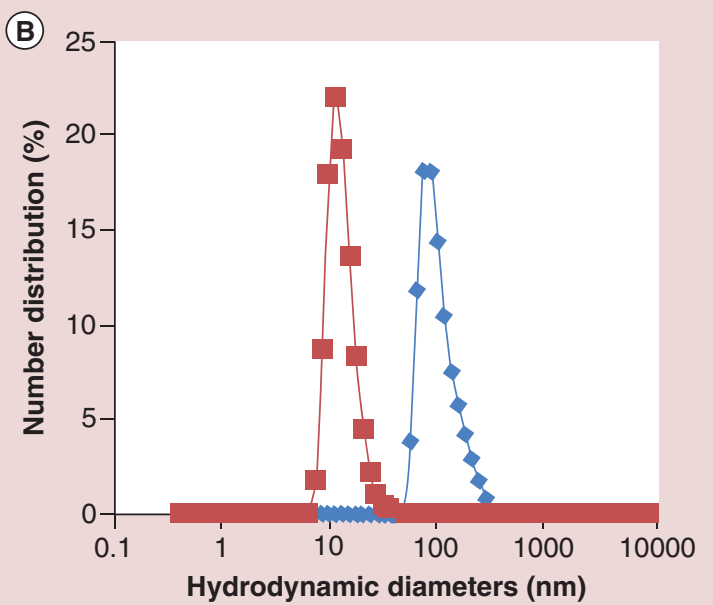

(C)
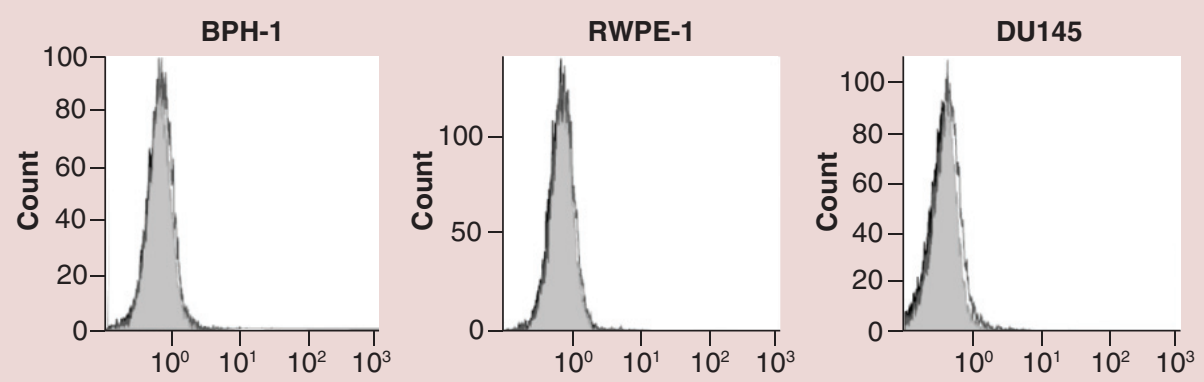

22RV1
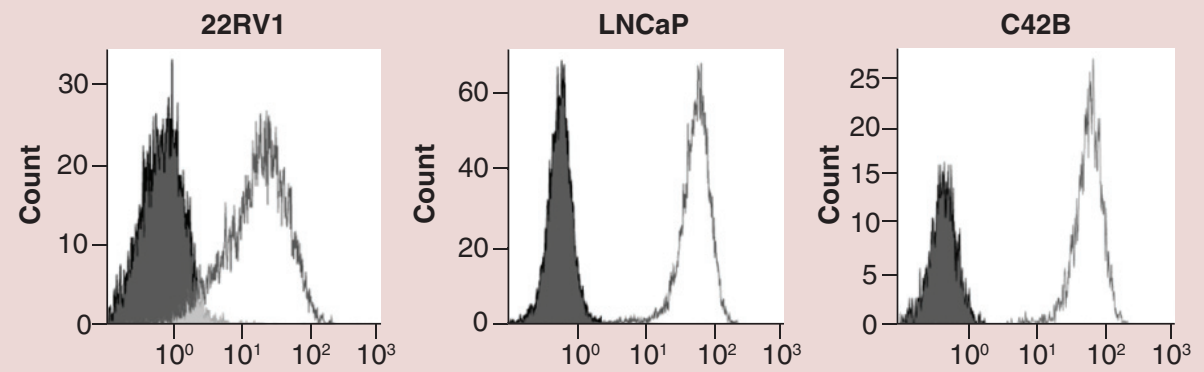

Figure 1. Characterization of iron oxide magnetic nanoparticles and J591 separately. (A) Transmission electron microscopy image of the magnetic nanoparticle (MNPs) as small aggregates. (B) Hydrodynamic diameters of the MNPs as synthesized (red line) and with the PEG-DSPE coating (blue line). (C) Flow cytometric analysis showed that the J591 antibody binds specifically to PSMA because positive staining was only detectable on PSMA-positive prostate cancer cells (22RV1, LNCaP and C42B) but not on PSMA-negative cells (BPH-1, RWPE-1, and DU145). For color figures, see online at www.futuremedicine.com/doi/full/10.2217/NNM.14.122 


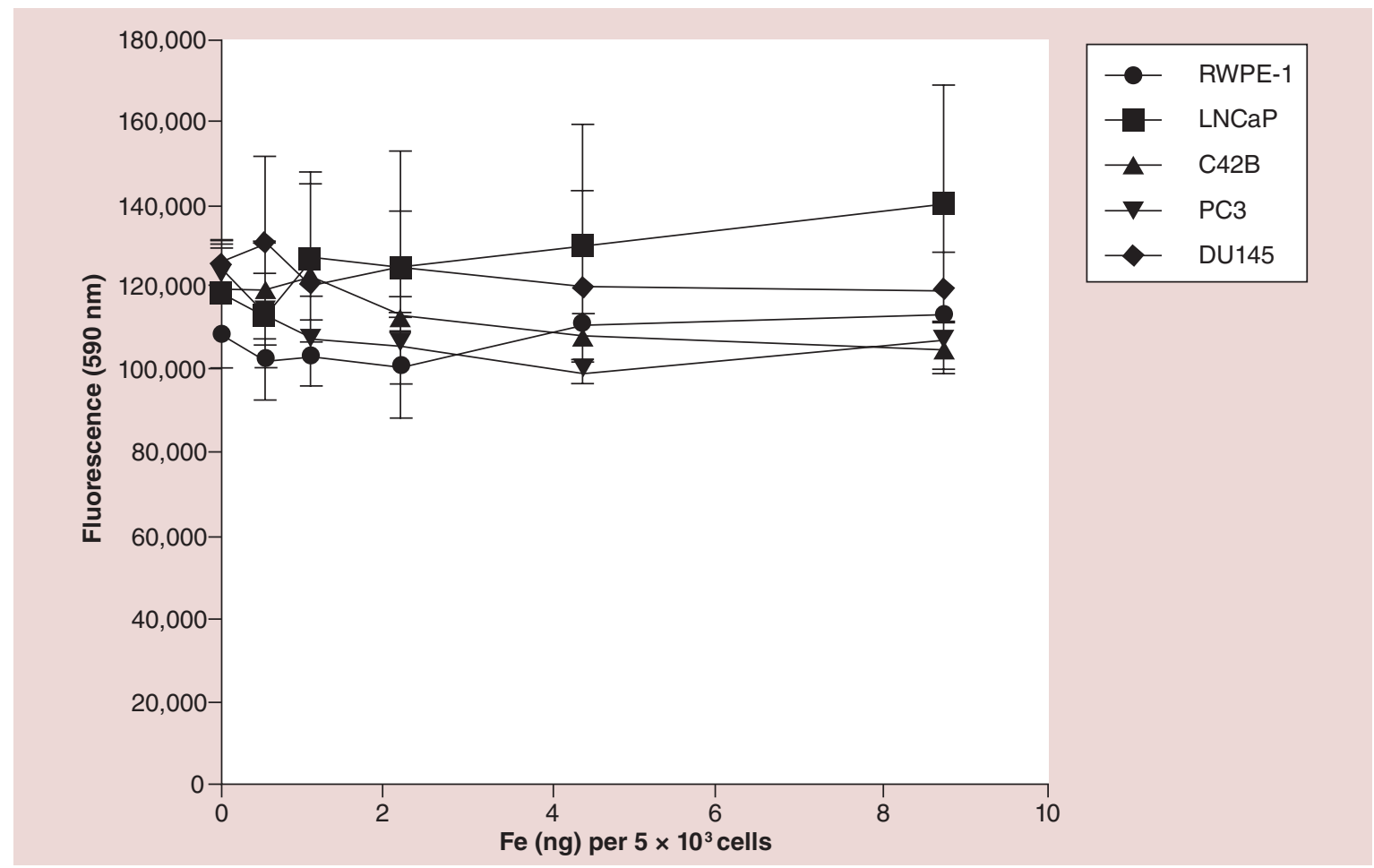

Figure 2. Magnetic nanoparticles are non-toxic to prostate cancer cells. Incubation of prostate cancer cell lines with various amounts of magnetic nanoparticles alone for $48 \mathrm{~h}$ did not affect their viability, as assessed by Alamar blue viability assay.

(Figure 6), suggesting improved tumor targeting by the conjugates. Iron uptake was strong in the spleens of all mice, independent of treatment group (Figure 6). A low level of iron uptake was seen in the liver of all mice, with no difference between the two groups.

\section{Discussion}

Prostate cancer is currently imaged using MRI, CT, TRUS and nuclear medicine [27]. However, these imaging techniques do not provide adequate detection for small tumor volumes or lymph node involvement [2]. The ability to accurately detect and locate small tumors is necessary for early detection of disease and for assessment of response to therapy in cancer patients. In recent years, the use of biomarker-targeted probes linked with nanoparticle-based contrast agents to enhance these imaging modalities has been a major area of research. In this study, proof-of-concept is provided that iron oxide MNPs conjugated with the PSMA-targeting antibody, J591, enhances MRI of prostate cancer in a preclinical model of orthotopic prostate cancer xenografts in mice.

MRI is a highly desirable imaging technology because it is non-invasive, provides excellent soft tissue contrast, multidimensional morphological information, and does not involve exposure to ionizing radiation [1]. MRI can be strongly enhanced through the employment of MNPs [1]. The presence of magnetic cores within the tissues decreases the relaxation times of the protons of surrounding water, causing a darkening effect at those sites in MR imaging. We showed that direct injection of MNPs into the prostate of mice (Figure 4) or intravenous injection of PSMA-targeting MNPs, which homed to the orthotopic tumors (Figure 5), caused darkening at those anatomical locations in MR images. These observations have major clinical implications because tumor-targeting MNPs could potentially enable the early detection of tumors confined within the prostate by MRI. Although PSMA is also expressed on normal prostate cells, the level is significantly lower compared with malignant cells. In addition, and very importantly, small metastases in lymph nodes and bone metastases may also be visualized using this technique. A key point is that lesions identified through this MNP-assisted MRI approach are prostate-derived. Currently, MRI and CT use lymph node size to distinguish between those that are malignant or benign, an approach based on the premise that the enlargement of nodes is due to the accumulation of metastatic cells [28]. However, not all enlarged lymph nodes contain tumor cells, and normal-sized lymph nodes can also harbor metastases, hence false-positives and false-negatives remain as limitations. MNP-enhanced MRI could potentially facilitate accurate nodal staging, independent of node size, an important prognostic factor [28]. Bone metastases are 
(A)

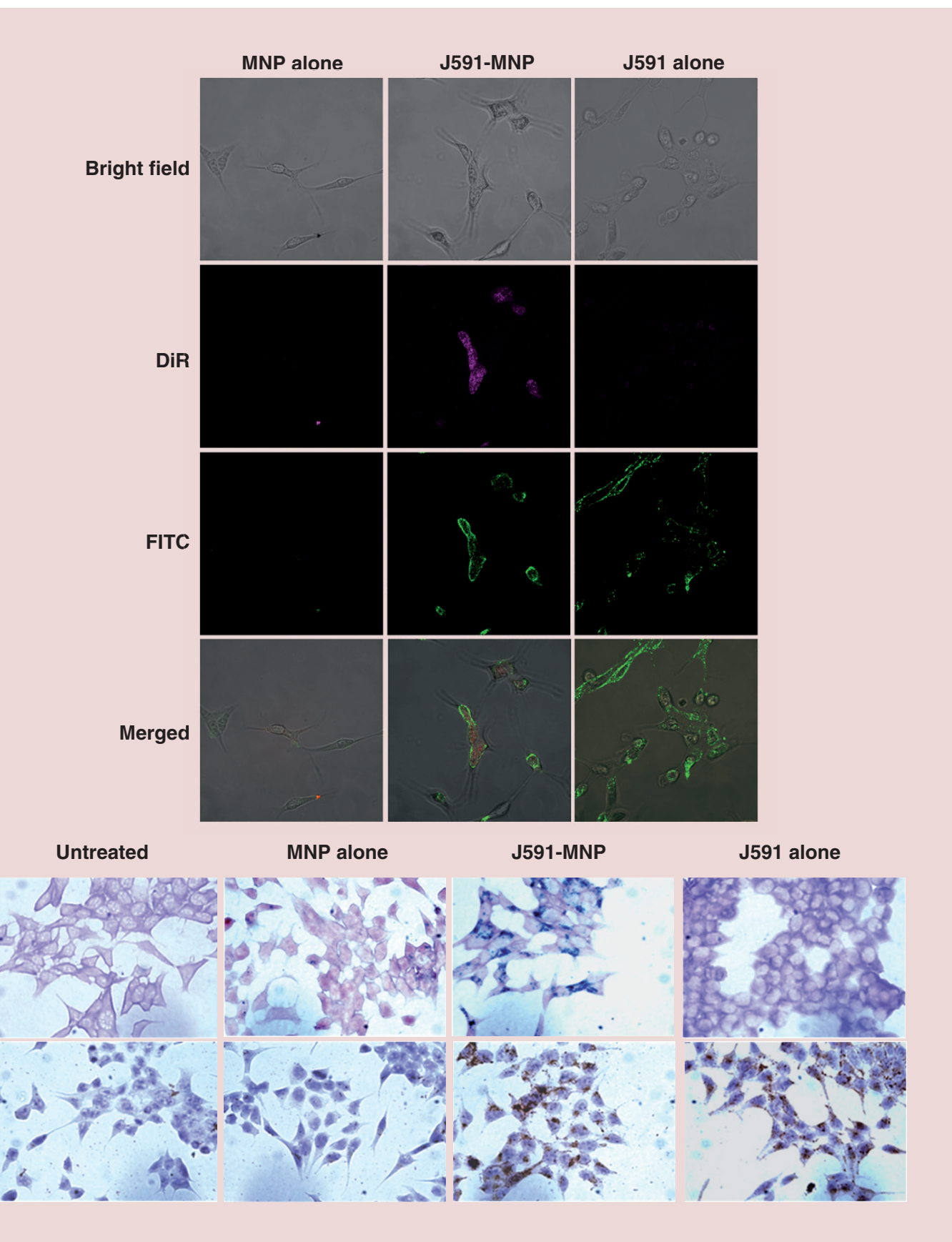

(B)

A)

Figure 3. J591-MNP led to increased tumor uptake of iron, and binding of J591 to PSMA is not compromised when conjugated to magnetic nanoparticles. (A) Confocal images of C42B cells after incubation with MNPs alone, J591-MNP or J591 alone, show that uptake of MNPs (DiR-labelled; purple) is increased when conjugated to J591. The J591 component of J591-MNP could be visualized with the FITC-labeled secondary antibody. Images were acquired on a Zeiss confocal microscope. (B) Prussian blue staining for iron (top panel; dark blue) showing greater iron uptake by LNCaP-LN3 cells with J591-MNP than MNP alone. Immunohistochemical staining for PSMA (bottom panel) shows that both J591-MNP, and J591 alone, give the same level of staining on LNCaP-LN3 cells, indicating that conjugation of MNPs to J591 does not affect its binding and specificity for PSMA. Minimal staining was seen with MNP alone.

MNP: Magnetic nanoparticle.

currently identified through radionuclide bone scans, and in the future this approach could be complemented with MNP-enhanced MRI.
An important consideration in the design of biomarker-targeting imaging probes is the biological characteristics of the biomarker itself. PSMA is an 


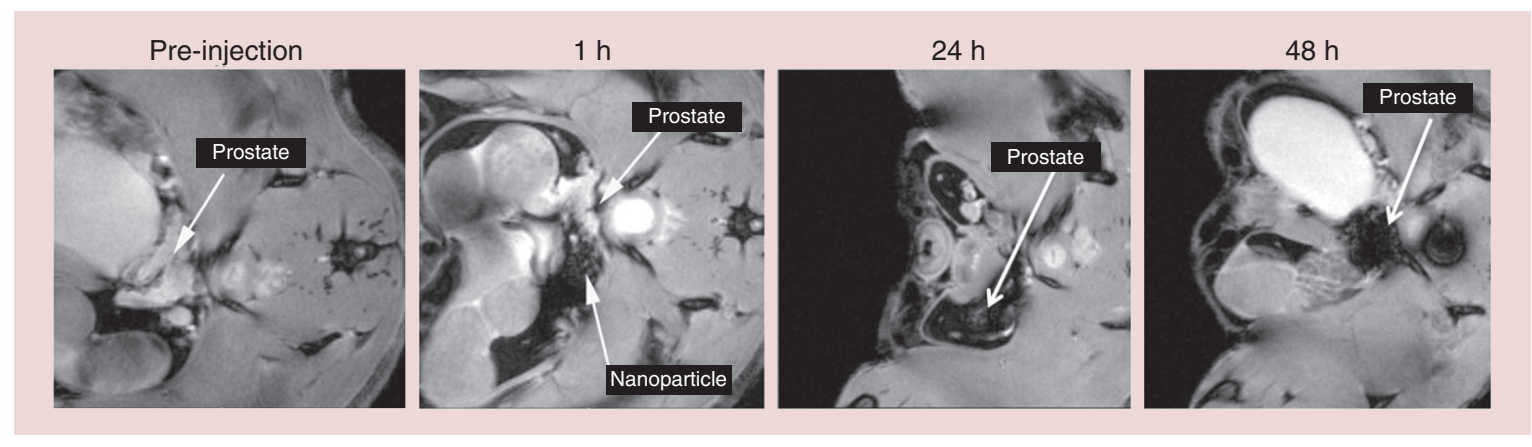

Figure 4. Direct injection of magnetic nanoparticles into the prostate of non-tumor bearing mice causes darkening of magnetic resonance images of the prostate. The prostate appears white/pale in magnetic resonance (MR) images taken prior to magnetic nanoparticle (MNP) injection, but it appears black and granulated on MR images once MNP was injected (12 $\mu \mathrm{g}$ of MNP in $20 \mu$ l volume). This negative contrast effect was seen 1-, 24- and 48-h post MNP injection in the same live mouse. MR images of a representative mouse are shown.

excellent prostate tumor biomarker to target for a number of reasons. Its expression is highly restricted to prostatic cells, although it has also been reported to be expressed on endothelial cells, and its level increases with higher cancer stages [29]. Unlike PSA, the clinically used biomarker of prostate cancer, PSMA, is still expressed on tumors from patients undergoing androgen deprivation therapy. But very importantly, the cytoplasmic domain of PSMA contains a novel motif of amino acid sequence MXXXL, that mediates its internalization and endosomal recycling via a clathrin-dependent mechanism [13,14]. This leads to high intracellular uptake of ligands carrying nanoparticles such as MNPs or other compounds, an important quality that improves tumor imaging due to the retention of the contrast agent for longer duration. Indeed, it was found that the MNP-mediated darkening effect on MR images lasted for at least $24 \mathrm{~h}$ in live mice (Figure 5). The specificity of the targeting agent is also very important in the design of biomarker-targeting imaging probes. In this study, we used the 5591 monoclonal antibody due to its well established specificity. ${ }^{177} \mathrm{Lu}-\mathrm{J} 591$ was been previously shown in a phase II clinical trial to target all known sites of disease in all treated subjects [15]. In another study, treatment of patients with ${ }^{111} \mathrm{In}-\mathrm{J} 591$ and then with ${ }^{90} \mathrm{Y}-\mathrm{J} 591$ revealed $89 \%$ of known bony metastases and $69 \%$ of soft tissue lesions [16]. The high specificity of J591 for PSMA was also confirmed by us using flow cytometric analysis of a panel of prostate cancer cell lines with known PSMA expression status (Figure 1B). When J591 was conjugated with MNPs, the binding ability of the antibody to PSMA was not compromised (Figure 3), which collectively suggests that this imaging platform is stable, and shows promise for translation into the clinic. MNPs also have the potential to be easily adapted to other imaging technologies such as nuclear or optical modalities, to provide anatomical, functional and molecular information [3].

Another major issue in the translation of nanotechnology into biomedical practice is the biocompatibility of the nanoparticles. MNPs have been demonstrated to be biocompatible and biodegradable [1]. It is suggested that following internalization into cells, iron oxide cores degrade into iron ions which then incorporate into the hemoglobin pool [30]. In addition, coating of MNPs with biodegradable polymers such as dextran or PEG prevents agglomeration, which also improves biocompatibility [3]. In this study, MNPs did not affect the viability of a panel of prostate cell lines, including both benign and cancerous cell lines (Figure 2), and the well-being of mice $24 \mathrm{~h}$ after MNP injection was not compromised (no changes to body weight, reflex, alertness, breathing rates, coating appearance, fecal texture and so on, as compared with pre-injection; see Materials \& methods for details). Currently, ferumoxil (GastroMARK), an iron oxide-based MNPs formulation, is approved for use in clinical MRI to enhance imaging of the bowel. However, there have been some reports of unwanted side effects in MNP usage. Feridex, a formerly US FDA-approved MNP agent administrated intravenously for imaging of the reticuloendothelial system that is based on the engulfment of MNPs by phagocytes, is known to cause back and groin pain, as well as inducing allergic reactions [31]. It is inevitable that intravenously injected MNPs, even those labelled with ligands to bind cell surface markers on cancer cells, will distribute to other body parts such as spleen and liver. From a clinical point of view, it is crucial to modify the MNPs (e.g., PEGylation) to limit their toxicity, and direct them to the anatomical site of interest via the attachment of a ligand to a cellsurface tumor biomarker to improve imaging at that site. If efficient targeting is achieved, a lower overall dose could be used to create a good MRI signal, and 


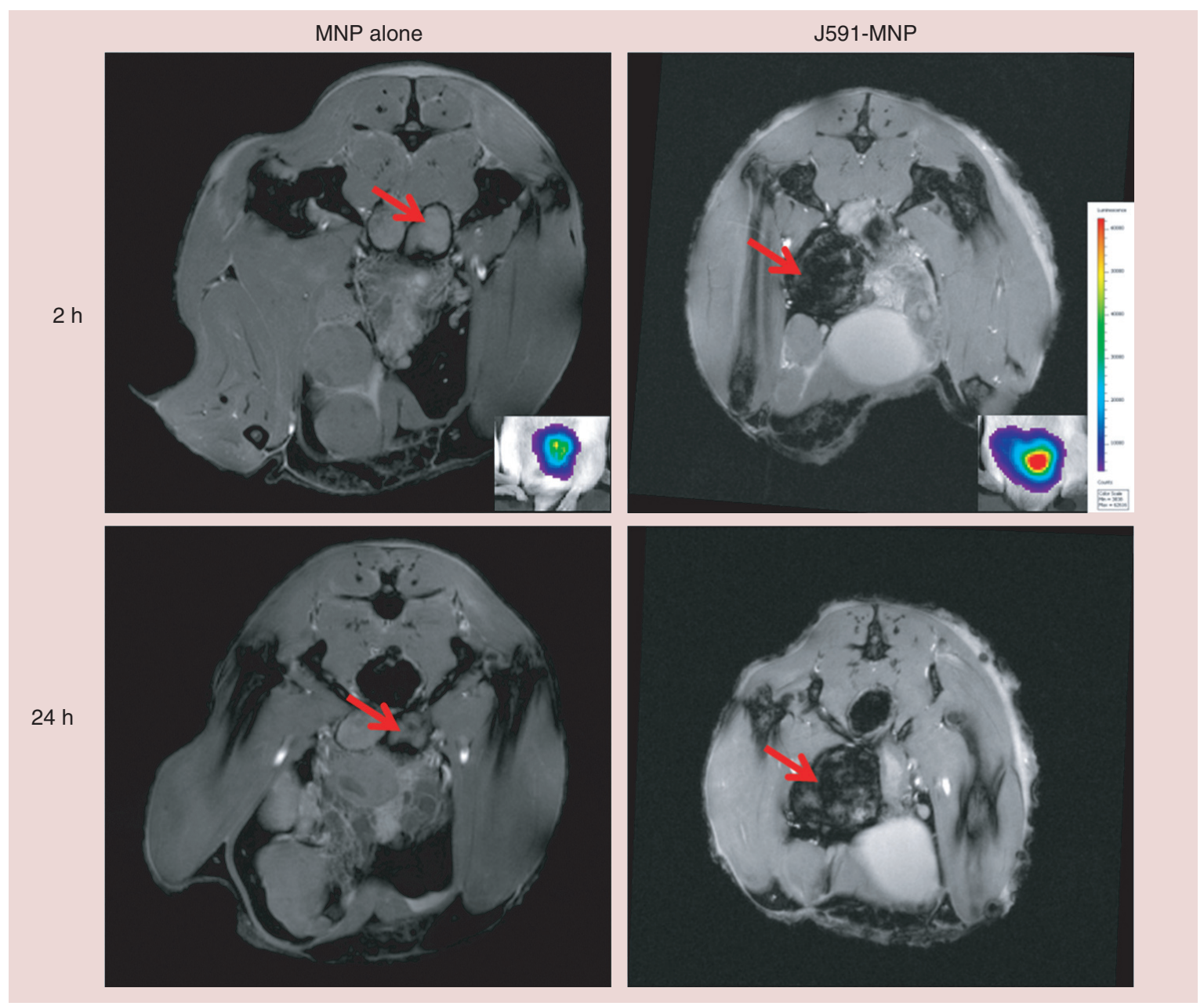

Figure 5. Enhancement of MRI with J591-MNP (pilot study). Once the pre-established LNCaP-luc tumors had reached the desired size (based on bioluminescence signal; inset), which occurred approximately 4-weeks post tumor cell injection, the mice were injected intravenously with MNPs alone $(n=2)$ or J591-MNP $(n=3)$. Administration of J591-MNP conjugates resulted in significant darkening of magnetic resonance images of the prostate region, at 2- and 24-h post-injection. No darkening effect occurred in mice given MNPs alone. Red arrows: orthotopic LNCaP-luc tumors. Magnetic resonance images of representative mice from each group are shown. MNP: Magnetic nanoparticle.

therefore with lower potential side effects. It was found that when mice were injected with the targeted MNPs, there was high iron uptake by the tumor, in contrast to those given non-targeted MNPs, which had negligible uptake (Figure 6). This correlated with the enhanced imaging (negative contrast) of orthotopic tumors by J591-MNP (Figure 5). None of the tumors from either group were hemorrhagic, further confirming that the stronger signals seen in J591-MNP tumors were indeed due to greater MNP uptake from PSMA-targeting, and not from them being hemorrhagic (higher iron content). It was also found that iron uptake was high in the spleens of mice, with no difference in the level between the two treatment groups (Figure 6). This finding is not surprising as only a small percentage of PSMA-targeting MNPs is expected to accumulate within the tumor, with the remaining distributing through the whole body. There was also no difference in liver iron uptake between targeting and non-targeting MNPs groups (Figure 6), likely for the same reason. The level of uptake in the liver was lower than in the spleen, which is an intriguing finding since preferential liver uptake is usually observed for nanoparticles after intravenous administration. It might be related to the physicochemical properties of the phospholipid coated nanoparticles used in this study [32] and warrants further investigation.

The use of nanoparticles as contrast agents for MRI of prostate cancer has been investigated by other groups as well. Abdolahi et al. used a similar approach to this one in that J591 was conjugated to MNPs, and showed that this complex enhanced MRI of LNCaP (PSMAproficient) but not DU145 (PSMA-deficient) cells [33]. These results are consistent with the data presented 


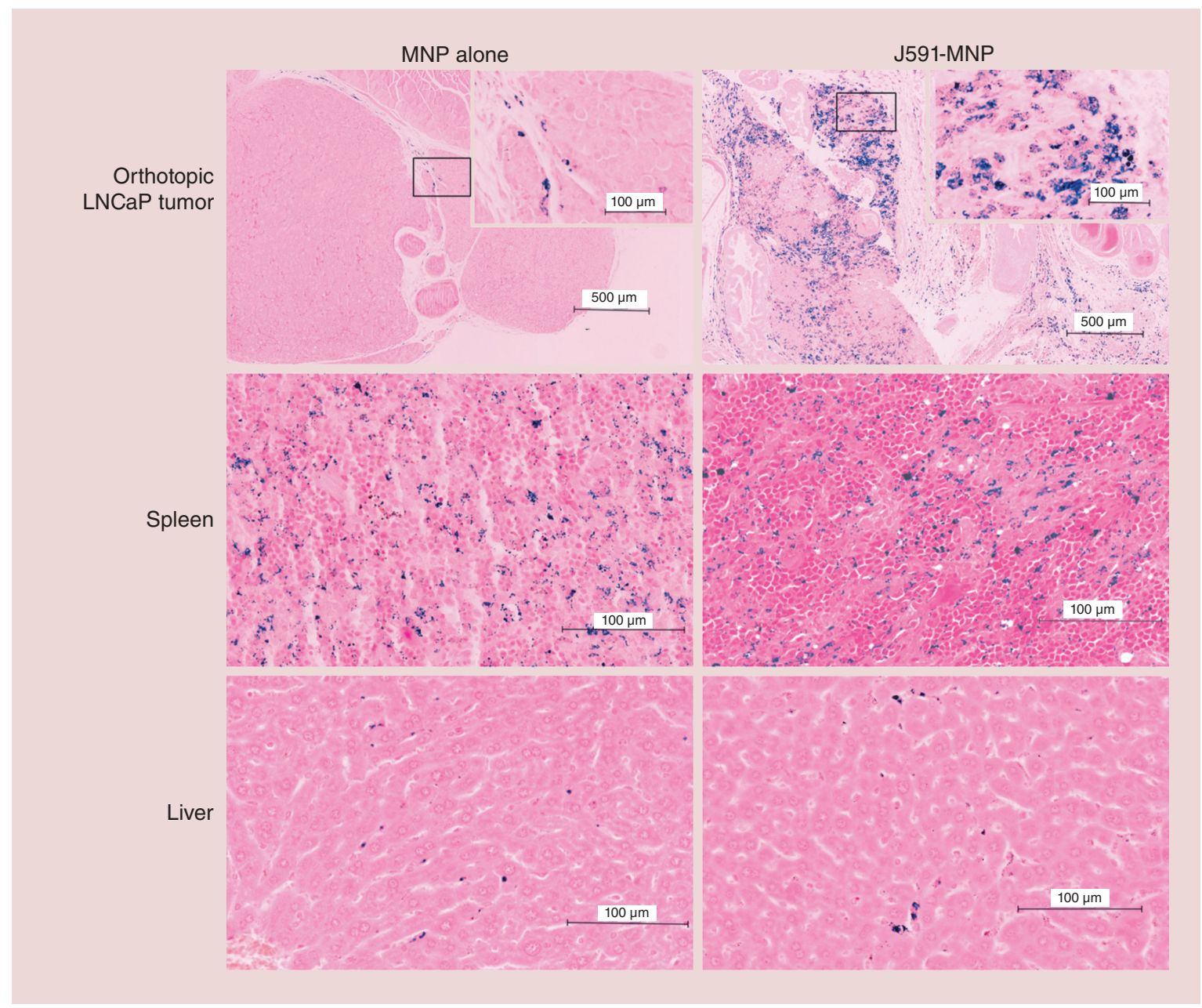

Figure 6. Targeting of orthotopic tumors is improved when J591 is conjugated to magnetic nanoparticles.

Prussian blue staining showed significant iron uptake in resected LNCaP tumors from mice injected intravenously with J591-MNP $(n=5)$, as compared to MNP alone $(n=4)$. Similar high levels of iron uptake is observed in the spleens between J591-MNP and MNP-alone mice. Low iron uptake was seen in liver from all mice. MNP: Magnetic nanoparticle.

here, although their study was limited to in vitro work only. Tan et al. reported that conjugation of CLTI peptide, which is specific to fibrin-fibronectin complexes in stroma, provided MRI contrast enhancement of subcutaneous PC3 xenografts in mice [34]. While this approach did not target prostate cancer cells per se, it still effectively enhanced MR detection of these tumors with good contrast-to-noise ratio. Gao et al. designed a theranostics technology for prostate cancer, whereby MNPs conjugated with docetaxel and a singlechain antibody against prostate stem cell antigen [35]. When injected in mice bearing PC3M xenografts, the nanoparticles provided MRI negative contrast, as well as inhibiting tumor growth and prolonging their survival. Together with the promising data presented here, this body of work confirms the relevance of immunotargeting superparamagnetic nanoparticles towards improving MR imaging of prostate cancer.

\section{Conclusion}

This study provides proof-of-concept that PSMA-targeted MNPs can effectively enhance MRI of prostate cancer in a preclinical model of the disease. Based on its biocompatibility, stability, together with its ability to enhance MRI, PSMA-targeting MNPs have promise to be translated into the clinic to improve the management of prostate cancer.

\section{Future perspective}

MRI will continue to be a major imaging tool for prostate cancer over the next decade due to its many desirable features as discussed above. As there has been an explosion in interest in the application of nanotechnology to improve imaging methods in recent years, we anticipate that some of the exciting and innovative strategies currently in development will be translated into the clinic in the short- to medium-term future, and 
indeed improve the management of cancer. In particular, strategies involving the use of biomarker-targeted probes linked with nanoparticle-based contrast agents to enhance cancer imaging have great potential for diagnosis, staging (lymph node involvement) and for real-time imaging of treatment response.

\section{Acknowledgements}

The authors wish to thank S Johnson for technical assistance with some experiments.

Financial \& competing interests disclosure

This project has been supported by Cancer Australia and Prostate Cancer Foundation of Australia (NHMRC-510239 and PG-
0409). The authors have no other relevant affiliations or financial involvement with any organization or entity with a financial interest in or financial conflict with the subject matter or materials discussed in the manuscript apart from those disclosed.

No writing assistance was utilized in the production of this manuscript.

\section{Ethical conduct of research}

The authors state that they have obtained appropriate institutional review board approval or have followed the principles outlined in the Declaration of Helsinki for all human or animal experimental investigations. In addition, for investigations involving human subjects, informed consent has been obtained from the participants involved.

\section{Executive summary}

\section{Current status of prostate cancer imaging in the clinic}

- MRI is routinely used for imaging of prostate cancer; however, improvements in tumor specificity and sensitivity are required especially for small tumors.

Development of a novel contrast agent to enhance MRI of prostate cancer

- PSMA is a cell surface membrane protein expressed highly on prostate cancer but minimally on normal prostate tissue (ideal imaging target).

- The superparamagnetic effect of iron oxide magnetic nanoparticles (MNPs) provides negative contrast in MRI.

- In this study, we conjugated the PSMA-specific antibody, J591, to MNPs. The antibody delivers the MNP specifically to prostate cancer cells, while the MNP component provides negative contrast in MRI.

Key findings of the authors' nanoparticle-antibody system for MRI

- MNPs did not affect the viability of prostate cancer cells per se, and were well tolerated by mice after injection.

- Significantly greater tumor uptake of MNPs occurred when they were labelled with J591, both in vitro and in vivo, than non-targeted MNPs.

- Importantly, J591-labelled MNPs localized to pre-established orthotopic tumors in mice, enhancing their detection by MR. High accumulation of iron was seen in these tumors; negligible accumulation for control group.

\section{The big picture}

- This system has the potential to enhance prostate cancer detection and localization in real-time, improving patient management.

\section{References}

1 Yigit MV, Moore A, Medarova Z. Magnetic nanoparticles for cancer diagnosis and therapy. Pharm. Res. 29(5), 1180-1188 (2012).

2 Taneja SS. Imaging in the diagnosis and management of prostate cancer. Rev. Urol. 6(3), 101-113 (2004).

3 Liu G, Gao J, Ai H, Chen X. Applications and potential toxicity of magnetic iron oxide nanoparticles. Small 9(9-10), 1533-1545 (2013).

4 Pouliquen D, Le Jeune JJ, Perdrisot R, Ermias A, Jallet P. Iron oxide nanoparticles for use as an MRI contrast agent: pharmacokinetics and metabolism. Magn. Reson. Imaging 9(3), 275-283 (1991).

5 To SY, Castro DJ, Lufkin RB, Soudant J, Saxton RE. Monoclonal antibody-coated magnetite particles as contrast agents for MR imaging and laser therapy of human tumors. J. Clin. Laser Med. Surg. 10 (3), 159-169 (1992).
6 Mahmoudi M, Serpooshan V, Laurent S. Engineered nanoparticles for biomolecular imaging. Nanoscale 3(8), 3007-3026 (2011).

7 Israeli RS, Powell CT, Fair WR, Heston WD. Molecular cloning of a complementary DNA encoding a prostate-specific membrane antigen. Cancer Res. 53(2), 227-230 (1993).

8 Grauer LS, Lawler KD, Marignac JL, Kumar A, Goel AS, Wolfert RL. Identification, purification, and subcellular localization of prostate-specific membrane antigen PSM' protein in the LNCaP prostatic carcinoma cell line. Cancer Res. 58(21), 4787-4789 (1998).

9 Schmittgen TD, Teske S, Vessella RL, True LD, Zakrajsek BA. Expression of prostate specific membrane antigen and three alternatively spliced variants of PSMA in prostate cancer patients. Int. J. Cancer 107(2), 323-329 (2003).

10 Wright GL Jr, Grob BM, Haley C et al. Upregulation of prostate-specific membrane antigen after androgendeprivation therapy. Urology 48(2), 326-334 (1996). 
11 Ghosh A, Heston WD. Tumor target prostate specific membrane antigen (PSMA) and its regulation in prostate cancer. J. Cell. Biochem. 91(3), 528-539 (2004).

12 Wright GL Jr, Haley C, Beckett ML, Schellhammer PF. Expression of prostate-specific membrane antigen in normal, benign, and malignant prostate tissues. Urol. Oncol. 1(1), 18-28 (1995).

13 Liu H, Rajasekaran AK, Moy P et al. Constitutive and antibody-induced internalization of prostate-specific membrane antigen. Cancer Res. 58(18), 4055-4060 (1998).

14 Rajasekaran SA, Anilkumar G, Oshima E et al. A novel cytoplasmic tail MXXXL motif mediates the internalization of prostate-specific membrane antigen. Mol. Biol. Cell 14(12), 4835-4845 (2003).

15 Bander NH, Milowsky MI, Nanus DM, Kostakoglu L, Vallabhajosula S, Goldsmith SJ. Phase I trial of ${ }^{177}$ lutetiumlabeled J591, a monoclonal antibody to prostate-specific membrane antigen, in patients with androgen-independent prostate cancer. J. Clin. Oncol. 23(21), 4591-4601 (2005).

16 Milowsky MI, Nanus DM, Kostakoglu L, Vallabhajosula S, Goldsmith SJ, Bander NH. Phase I trial of yttrium-90labeled anti-prostate-specific membrane antigen monoclonal antibody $\mathrm{J} 591$ for androgen-independent prostate cancer. J. Clin. Oncol. 22(13), 2522-2531 (2004).

17 Pinna N, Grancharov S, Beato P, Bonville P, Antonietti M, Niederberger M. Magnetite Nanocrystals: Nonaqueous Synthesis, Characterization, and Solubility. Chem. Mater. 17(11), 3044-3049 (2005).

18 Thierry B, Al-Ejeh F, Brown M, Majewski P, Griesser H.J. Immunotargeting of Functional Nanoparticles for MRI detection of Apoptotic Tumor Cells. Adv. Mater. 21, 541-545 (2009).

19 Thalmann GN, Anezinis PE, Chang SM et al. Androgenindependent cancer progression and bone metastasis in the LNCaP model of human prostate cancer. Cancer Res. 54(10), 2577-2581 (1994).

20 Wu TT, Sikes RA, Cui Q et al. Establishing human prostate cancer cell xenografts in bone: induction of osteoblastic reaction by prostate-specific antigen-producing tumors in athymic and SCID/bg mice using LNCaP and lineagederived metastatic sublines. Int. J. Cancer 77(6), 887-894 (1998).

21 Liu H, Moy P, Kim S et al. Monoclonal antibodies to the extracellular domain of prostate-specific membrane antigen also react with tumor vascular endothelium. Cancer Res. 57(17), 3629-3634 (1997).

22 Chen H, Wang L, Yu Q et al. Anti-HER2 antibody and ScFvEGFR-conjugated antifouling magnetic iron oxide nanoparticles for targeting and magnetic resonance imaging of breast cancer. Int. J. Nanomed. 8, 3781-3794 (2013).

23 Schleich N, Sibret P, Danhier P et al. Dual anticancer drug/superparamagnetic iron oxide-loaded PLGA-based nanoparticles for cancer therapy and magnetic resonance imaging. Int. J. Pharm. 447(1-2), 94-101 (2013).

$24 \mathrm{Bu} \mathrm{L}$, Xie J, Chen $\mathrm{K}$ et al. Assessment and comparison of magnetic nanoparticles as MRI contrast agents in a rodent model of human hepatocellular carcinoma. Contrast Media Mol. Imaging 7(4), 363-372 (2012).

25 Morton DB. Humane endpoints in animal experiments for biomedical research: ethical, legal and practical aspects. Hendriksen CFM, Morton DB (Eds). Royal Society of Medicine Press. London, England p 5-12 (1999).

26 Dubertret B, Skourides P, Norris DJ, Noireaux V, Brivanlou $\mathrm{AH}$, Libchaber A. In vivo imaging of quantum dots encapsulated in phospholipid micelles. Science 298(5599), 1759-1762 (2002).

27 Kelloff GJ, Choyke P, Coffey DS. Challenges in clinical prostate cancer: role of imaging. AJR Am. J. Roentgenol. 192(6), 1455-1470 (2009).

28 Harisinghani M. Nanoparticle-enhanced MRI: are we there yet? Lancet Oncol. 9(9), 814-815 (2008).

29 Osborne JR, Akhtar NH, Vallabhajosula S, Anand A, Deh K, Tagawa ST. Prostate-specific membrane antigen-based imaging. Urol. Oncol. 31(2), 144-154 (2013).

30 Thorek DL, Chen AK, Czupryna J, Tsourkas A. Superparamagnetic iron oxide nanoparticle probes for molecular imaging. Ann. Biomed. Eng. 34(1), 23-38 (2006).

31 Colombo M, Carregal-Romero S, Casula MF et al. Biological applications of magnetic nanoparticles. Chem. Soc. Rev. 41(11), 4306-4334 (2012).

32 Bertrand N, Leroux JC. The journey of a drug-carrier in the body: an anatomo-physiological perspective. J. Control. Release 161(2), 152-163 (2012).

33 Abdolahi M, Shahbazi-Gahrouei D, Laurent S et al. Synthesis and vitro evaluation of MR molecular imaging probes using J591 mAb-conjugated SPIONs for specific detection of prostate cancer. Contrast Media Mol. Imaging 8(2), 175-184 (2013).

34 Tan M, Burden-Gulley SM, Li W et al. MR molecular imaging of prostate cancer with a peptide-targeted contrast agent in a mouse orthotopic prostate cancer model. Pharm. Res. 29(4), 953-960 (2012).

35 Gao X, Luo Y, Wang Y et al. Prostate stem cell antigentargeted nanoparticles with dual functional properties: vivo imaging and cancer chemotherapy. Int. J. Nanomed. 7 , 4037-4051 (2012). 